\title{
Red Blood Cell Lifespan Shortening in Patients with Early-Stage Chronic Kidney Disease
}

\author{
Jiu-Hong $\mathrm{Li}^{\mathrm{a}}$ Jun-Feng Luo ${ }^{\mathrm{b}}$ Ying Jiang ${ }^{\mathrm{a}}$ Yong-Jian Ma ${ }^{\mathrm{b}}$ \\ Yong-Qiang Ji ${ }^{d}$ Guo-Liang Zhu ${ }^{d}$ Cong Zhou ${ }^{d}$ Hong-Wei Chu ${ }^{b}$ \\ Hou-De Zhang c \\ aDepartment of Nephrology, Nanshan Hospital, Guangdong Medical University, Shenzhen, \\ China; ${ }^{b}$ Guangdong Breath Test Engineering and Technology Research Center, Shenzhen \\ University, Shenzhen, China; ' Department of Gastroenterology, Nanshan Hospital, \\ Guangdong Medical University, Shenzhen, China; ${ }^{\mathrm{d}}$ Institute of Seekya Breath Test \\ Technology, Shenzhen, PR China
}

\author{
Keywords \\ Chronic kidney disease · Red blood cell lifespan - Renal anemia · Erythropoietin · \\ Levitt's CO breath test
}

\begin{abstract}
Background: Although reduced red blood cell (RBC) lifespan has been reported to be a contributory factor to anemia in patients with end-stage chronic kidney disease (CKD), there are limited data regarding RBC lifespan in early-stage CKD. Serum erythropoietin (EPO) is considered a primary causative factor of renal anemia. The aims of this study were to compare the RBC lifespan, serum EPO levels, and other renal anemia indicators across CKD-stage groups of patients and to analyze the impacts of etiological factors on renal anemia. Methods: A cohort of 74 non-smoking patients with CKD were enrolled, including 15 in stage 1, 18 in stage 2, 15 in stage 3, 15 in stage 4, and 11 in stage 5. RBC lifespan was determined by CO breath tests. Potential correlations of hemoglobin $(\mathrm{Hb})$ concentration with RBC lifespan, reticulocyte count (Ret), and levels of EPO, ferritin, folic acid, and vitamin B12 were analyzed. Results: CKD progression was associated with decreases in $(\mathrm{Hb})$ and RBC lifespan. RBC lifespan durations in CKD stages 1-5 were $122 \pm 50,112 \pm 26,90 \pm 32,88 \pm 28$, and $60 \pm 24$ days, respectively. RBC lifespan means for the stage 3, 4 and 5 groups were significantly shorter than those for the stage 1 and 2 groups. Serum EPO did not differ significantly between the CKD stage
\end{abstract}




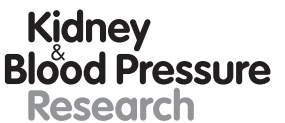

Research

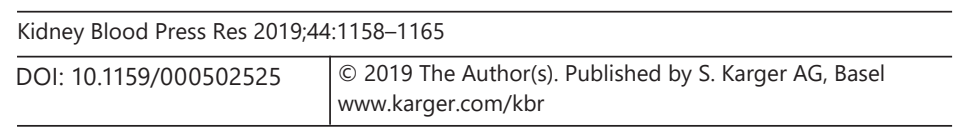

Li et al.: RBC Lifespan Shortening in Patients with Early-Stage CKD

groups. ( $\mathrm{Hb})$ correlated directly with RBC lifespan $(r=0.372, p=0.002)$ and Ret $(r=0.308$, $p=0.011$ ), but did not correlate with serum EPO, ferritin, folic acid, or vitamin B12 levels. Conclusions: Reduced RBC lifespan in early-stage CKD, demonstrated in this study, suggests that increased RBC destruction may play a more important etiological role in renal anemia than other indicators in patients with CKD.

(C) 2019 The Author(s)

Published by S. Karger AG, Basel

\section{Introduction}

Anemia is a common complication of chronic kidney disease (CKD) that tends to worsen with CKD progression [1]. The development of renal anemia has been attributed to multiple processes including erythropoietin (EPO) deficiency, suppression of bone marrow function by uremic toxins, iron deficiency and other nutritional deficits, bleeding, infection, underlying hematological disease, hyperparathyroidism, nutritional deficits, and an abnormally short red blood cell (RBC) lifespan [2,3]. Inadequate EPO production for maintaining erythropoiesis has been considered a primary etiologic factor of renal anemia. Recombinant human EPO (rhuEPO) therapy has been very effective in improving renal anemia since 1989 . However, EPO resistance may prevent patients' hemoglobin $(\mathrm{Hb})$ levels from reaching target values even after receiving a high-dose rhuEPO regimen for 4-6 months. Moreover, high-dose rhuEPO increases the risk of cardiovascular disease and mortality [4-6], and can even promote cancer progression [7].

RBC lifespan has been shown to be abnormally short in patients with renal failure [8, 9]. RBC lifespan refers to the duration of time that RBCs survive in circulation after they are released from bone marrow. The commonly referenced normal human adult RBC lifespan of about 120 days was derived from the transfused allogeneic RBC survival time. However, monitoring of RBC lifespan has been complicated by the fact that established $\mathrm{RBC}$ lifespan measurement methods with radioactive isotope labeling are cumbersome and time-consuming. Moreover, prior studies of RBC lifespan with such techniques had small sample sizes and RBC lifespan data from patients in early-stage CKD are limited. Hence, the RBC lifespan shortening in association with renal anemia may be underestimated.

Based on the discovery that endogenous $\mathrm{CO}$ originates mainly from heme oxidation associated with Hb degradation following RBC rupture, Strocchi et al. [10] and Furne et al. [11] developed a simple, rapid, and accurate methodology, known as the CO breath test, for RBC lifespan determination. A newly developed automated instrument based on Levitt's CO breath test enables RBC measurements to be taken with ease in clinical settings [10]. The aim of the present study was thus to use this new automated CO breath test to examine RBC lifespan across CKD stages and to assess the utility of this assessment relative to other renal anemia indicators, such as EPO levels.

\section{Materials and Methods}

\section{Study Subjects}

A cohort of 74 non-smoking patients diagnosed with CKD was recruited from the Department of Nephrology at Shenzhen Nanshan Hospital, Guangdong Medical School, Shenzhen, China. The cohort comprised of 50 males (mean age \pm SD $48 \pm 15$ years) and 24 females (mean age \pm SD $45 \pm 16$ years). They were divided into the following 5 CKD-stage groups based on KDIGO guidelines: stage $1, n=15$; stage 2, $n=18$; stage $3, n=15$; stage 4 , 


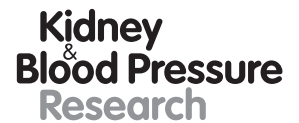

Kidney
Blood Pressure
Research

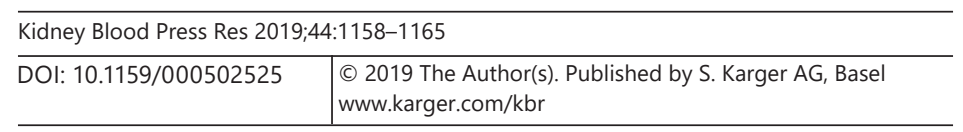

Li et al.: RBC Lifespan Shortening in Patients with Early-Stage CKD

$n=15$; and stage 5, $n=11$. The condition underlying CKD was IGA nephropathy in 19 individuals, diabetes mellitus in 17, chronic glomerulonephritis in 9, other factors in 29 individuals. The exclusion criteria included receiving rhuEPO, folic acid, chalybeate, and/or vitamin B12 treatments; clear sign of acute infection; and comorbidity diagnosis with a hemorrhagic disorder, chronic lung disease, or cancer. All of the patients provided written informed consent to participate in the study. The study protocol was approved by the Institutional Review Board of Nanshan Hospital.

\section{Indicators of Anemia}

On the same day that alveolar air sampling was performed, peripheral vein blood samples were collected for the detection of anemia indicators including RBC lifespan, ( $\mathrm{Hb})$, reticulocyte count (Ret), EPO levels, ferritin levels, folic acid levels, and vitamin B12 levels.

\section{RBC Lifespan}

In accordance with Levitt's CO breath test, the mean RBC lifespan was measured as the total capacity of $\mathrm{CO}$ from $\mathrm{Hb}$ degradation divided by the quantity of $\mathrm{CO}$ released per day. Alveolar air samples were collected in the morning without a fasting requirement. Briefly, after a deep inspiration, each subject held his or her breath for $10 \mathrm{~s}$, and then exhaled into the collection system through a mouthpiece.

The collection system discarded the first $300 \mathrm{~mL}$ of volume, which was considered to contain dead space gas, and then directed subsequent alveolar air automatically into a foil collection bag. If needed, the procedure was repeated until the collected air sample reached the collection bag's 1,000-mL capacity. The filled bag was detached and sealed immediately. Atmospheric samples were collected just after breath sampling. Alveolar air and atmospheric samples were stored at room temperature and analyzed within 5 days. The instrument used to determine RBC lifespan was the ELS Tester (Seekya Biotec Co., Ltd., Shenzhen, China), which measures endogenous $\mathrm{CO}$ by non-dispersive infrared comparison of the CO content within an alveolar air sample versus that in the accompanying atmospheric air sample using Levitt's formula [10]. Operation of this instrument involved a simple 3-step protocol: (1) addition of alveolar and environmental samples; (2) inputting of ( $\mathrm{Hb}$ ) information; and (3) pressing a start measurement button. The measurements and calculations for each assessment were completed within $15 \mathrm{~min}$.

\section{Kidney Function}

Estimated glomerular filtration rate (eGFR) was calculated according to the IDMScorrected simplified MDRD formula whereby MDRD-eGFR $=175 \times$ serum creatinine $(\mathrm{mg} /$ dL) $-1.154 \times$ age (years)-0.203. Serum creatinine was obtained from the laboratory archives which were measured within 2 days of the RBCs lifespan measurement.

\section{Statistical Analysis}

Normally distributed data, including RBC lifespan, (Hb), EPO level, and eGFR data, were given as means with SDs. Abnormally distributed data, including Ret, ferritin, folic acid, and vitamin B12 levels, were given as medians with interquartile ranges. Pearson and Spearman analyses were used to analyze correlations between normally and non-normally distributed datasets, respectively. Partial correlation analysis was used to detect independent correlations between variables. Paired $t$ tests and non-parametric tests were performed to compare data across CKD stage groups for normally and non-normally distributed data, respectively. The statistical analyses were conducted in SPSS 22.0 (SPSS for Windows, version 22, SPSS, Chicago, IL, USA). $p<0.05$ was considered significant. 


\section{Kidney \\ Blood Pressure \\ Research}

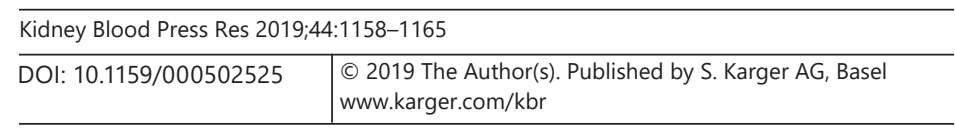

Li et al.: RBC Lifespan Shortening in Patients with Early-Stage CKD

\section{Results}

\section{Variable Differences Across CKD Stage Groups}

No effects of age or gender were observed. The mean RBC lifespans and data for 6 other indicators of anemia were reported for each CKD stage group in Table 1. (Hb) decreased with increasing CKD stage (Table 1). Meanwhile, a gradual reduction in RBC lifespan with advancing CKD stage was observed, as shown in Table 1 and Figure 1. The mean RBC lifespan obtained for the CKD stage 1 group was within normal range, while that obtained for the CKD stage 2 group was slightly below normal range, though the two did not differ significantly from each other. The mean RBC lifespans obtained for the CKD stage 3, 4, and 5 groups were significantly shorter than the values obtained for the CKD stage 1 and 2 groups $(p<0.05)$. Notably, the mean RBC lifespan obtained for the CKD stage 5 group was half the magnitude of the mean RBC lifespan obtained for the CKD stage 1 group.

Ret did not differ significantly among the CKD stage 1-4 groups, but was significantly shorter in the CKD stage 5 group than in the CKD stage 1 group $(p<0.05)$. Mean blood ferritin, folic acid, and vitamin B12 levels did not differ significantly between any of the CKD stage groups. Likewise, EPO did not differ significantly between any of the groups (Fig. 2).

\section{Variable Correlations with $(\mathrm{Hb})$}

Pearson bivariate correlation analyses revealed that $(\mathrm{Hb})$ correlated directly with eGFR $(r=0.550, p<0.001)$ and RBC lifespan $(r=0.476, p<0.001)$, but not with EPO levels $(p=$ $0.066)$. Spearman bivariate correlation analyses revealed that $(\mathrm{Hb})$ correlated directly with Ret $(r=0.435, p<0.001)$ and correlated inversely with folic acid $(r=-0.282, p=0.016)$ and vitamin B1 ( $r=-0.259, p=0.027$ ) levels. To control for the possibility that these correlations may be secondary to primary correlations with eGFR, partial correlation analysis was performed. That partial correlation analysis confirmed $(\mathrm{Hb})$ correlations with only RBC lifespan $(r=0.372, p=0.002)$ and $\operatorname{Ret}(r=0.308, p=0.011)$.

\section{Discussion}

In this study, we found that RBC lifespan shortening, which reflects the speed of RBC rupture and has been associated previously with CKD only at advanced CKD stages, was evident already in early-stage CKD patients. Ret, which reflects bone marrow hematopoietic function, was not significantly decreased until CKD stage 5. We did not find relationships of CKD stage with EPO, ferritin, folic acid, or vitamin B12 levels. Notably, our use of a highly efficient, automated RBC lifespan-measuring instrument [12] provided the benefit of enabling a larger sample size of participants to be analyzed relative to previous studies of RBC lifespan.

Fundamentally, anemia occurs when there is an imbalance between erythropoiesis and RBC degradation. In the present cohort of 74 CKD patients, RBC lifespan shortening emerged as a primary correlate of renal anemia, with other implicated factors, especially EPO levels, appearing to be less important or unimportant. These data indicate that the contribution of RBC rupture to the etiology of renal anemia should be further examined.

The correlation between RBC lifespan and eGFR suggests that there are unknown pathogenic factors at work during early-stage CKD that are associated with the decline of eGFR. In the 1960s, Loge et al. [13] and Desforges and Dawson [14] observed that when blood from uremic patients was transfused into recipients with normal blood urea levels, the recipients maintained a normal RBC lifespan, whereas when blood from normal donors was transfused into uremic patients, reductions in RBC lifespan were observed [8,9]. Hence, it appeared that the serum of the uremic patients contained some factor or factors that were hastening eryp- 
Fig. 1. RBC lifespan across CKD stages. * RBC lifespan is significantly shorter in stages 3,4 , and 5 than in stages 1 and $2(p<0.05)$. $\mathrm{RBC}$, red blood cell; CKD, chronic kidney disease.

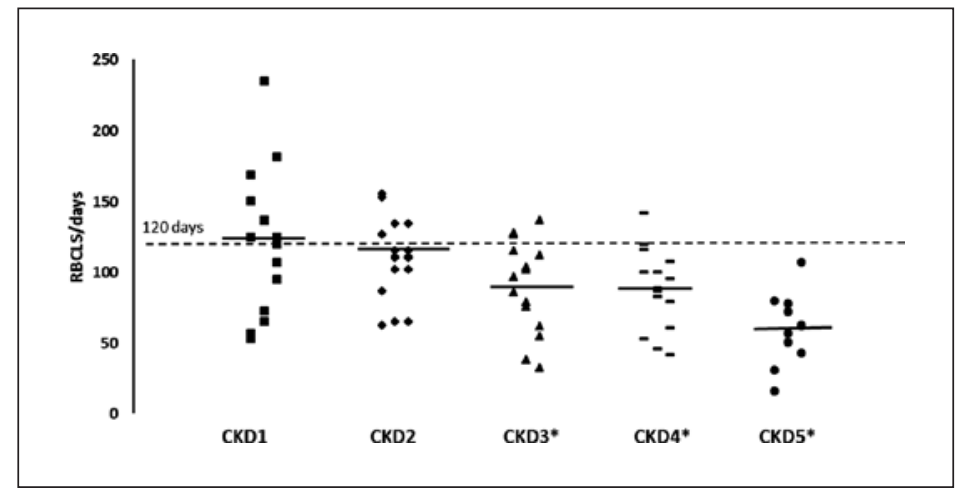

Table 1. Renal anemia indicator data by CKD stage group

\begin{tabular}{|c|c|c|c|c|c|}
\hline \multirow[t]{2}{*}{ Indicator } & \multicolumn{5}{|l|}{ CKD stage } \\
\hline & 1 & 2 & 3 & 4 & 5 \\
\hline $\mathrm{Hb}, \mathrm{g} / \mathrm{L}$ & $134 \pm 16$ & $146 \pm 20$ & $122 \pm 23^{*}$ & $99 \pm 20^{*}$ & $99 \pm 19 *$ \\
\hline RBC lifespan, days & $122 \pm 50$ & $112 \pm 26$ & $90 \pm 32 *$ & $88 \pm 28 *$ & $60 \pm 24^{*}$ \\
\hline EPO, IU/L & $9.0 \pm 4.1$ & $7.7 \pm 4.9$ & $11.1 \pm 5.7$ & $10.6 \pm 4.5$ & $7.2 \pm 4.7$ \\
\hline Ret $/ 10^{6} \mu \mathrm{L}$ & $0.07(0.05-0.07)$ & $0.06(0.05-0.09)$ & $0.07(0.04-0.1)$ & $0.05(0.04-0.07)$ & $0.04(0.03-0.05)^{*}$ \\
\hline Ferritin, ng/mL & $99(60-178)$ & 161 (109-329) & $283(143-448)$ & $149(79-378)$ & $203(115-390)$ \\
\hline Folic acid, ng/mL & $10.2(8.7-16.1)$ & $13.4(8.5-15.5)$ & $14.2(10.3-18.4)$ & $15.6(14.2-18.2)$ & $15.6(14.9-18.4)$ \\
\hline Vitamin B12, pg/mL & $402(364-541)$ & $434(415-503)$ & 517 (394-837) & $540(438-723)$ & $509(404-965)$ \\
\hline
\end{tabular}

* Significantly shorter than CKD stage $1(p<0.05)$.

RBC lifespan, serum EPO, and (Hb) data are means \pm SD. Serum ferritin, folic acid, vitamin B12, and Ret data are medians (interquartile ranges).

Hb, hemoglobin; CKD, chronic kidney disease; RBC, red blood cell; EPO, erythropoietin; Ret, reticulocyte count.

tosis or RBC rupture. Although micromolecule toxins in uremic patients' blood can be leached out in traditional hemodialysis, RBC lifespan of uremic patients is not improved by hemodialysis [15]. On the contrary, EPO-stimulating agent dosage requirements were reduced and anemia was corrected more effectively by online hemodialysis filtration, which clears medium-sized molecular and macromolecular toxins, than by traditional hemodialysis [16, 17]. Such findings suggest that the pathogenic factors leading to RBC lifespan shortening would be expected to be medium- to macro-sized molecules.

Potential candidates of interest include acrolein, indoxyl-sulfate, and several eryptosisstimulating molecules [18-20]. Further studies should be carried out to examine these possibilities.

Peripheral blood Ret is an indicator of bone marrow hematopoietic function that correlates with the rate of erythropoiesis. Normally, patients with hypoxia or anemia will have augmented bone marrow erythroid hematopoiesis, resulting in increased Ret, to alleviate anemia and stabilize peripheral ( $\mathrm{Hb})$. In the current study, we did not see significant compensatory elevation of Ret in CKD patients, even among those patients whose disease was in an advanced stage. Indeed, Ret was significantly decreased in the CKD stage 5 group compared to the CKD stage 1 group. 
Fig. 2. Serum EPO concentrations across CKD stages. No significant differences between the groups were detected ( $p>0.05)$. However, the curve shows a possible trend of a temporary earlystage compensatory increase. EPO, erythropoietin; CKD, chronic kidney disease.

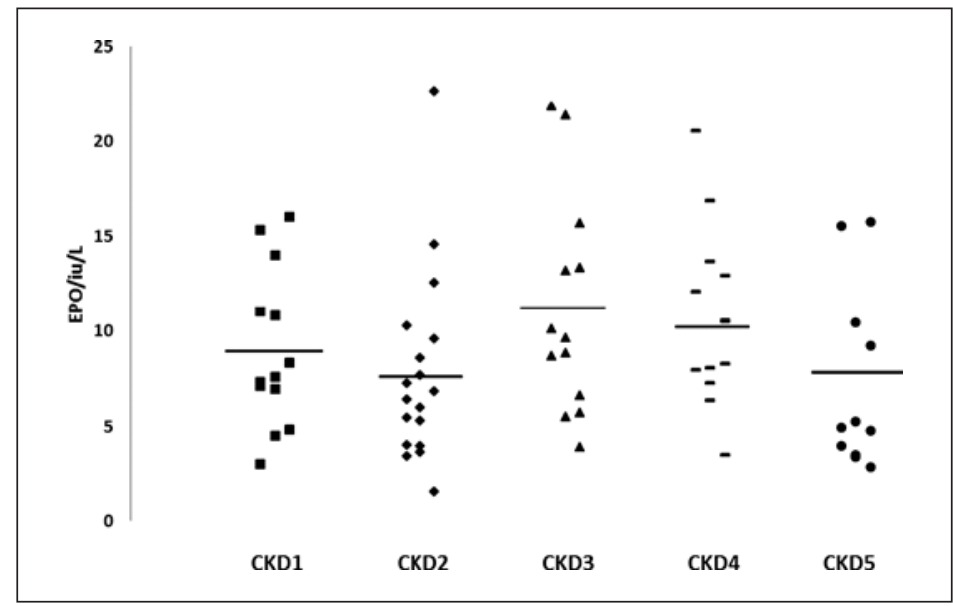

Hence, the compensative capacity of bone marrow appears to be weakened in anemic CKD patients, perhaps due to the inhibition of erythroid cell proliferation in bone marrow. Circulating polyamine toxins and/or cytokines in uremic patients have been suspected to inhibit RBC proliferation [21]. Of cytokines, fibroblast growth factor 23 is the most attractive. Recently, Shima et al. [22] confirmed that sucroferric oxyhydroxide decreases serum phosphorus level and fibroblast growth factor 23 and improves renal anemia in hemodialysis patients. Hence, suppression of bone marrow function can lead to renal anemia and may underlie EPO-stimulating agent therapy inefficacy.

Although inadequate EPO production has been postulated to be a primary factor in renal anemia [23], we did not find significant differences in EPO across CKD stages or a lack of EPO per se in our patients with CKD. Previous studies have described an elevation of EPO during early-stage CKD followed by a decrease [24]. Although there were no significant findings in this regard in this study, the shape of the EPO concentration curve relative to advancing CKD stage, shown in Figure 2, was consistent with such a pattern. Given that EPO synthesis and secretion is regulated in response to hypoxia and anemia, it is reasonable to suppose that EPO elevation in CKD patients' sera reflects an attempt to meet the increased demands for new RBC generation created by increased RBC destruction and decreased (Hb). However, this compensatory mechanism is limited and, ultimately, insufficient in patients who suffer kidney failure. Regardless, the present findings indicate that EPO deficiency is not an essential factor of renal anemia in early-stage CKD.

Renal anemia has been associated with abnormalities of the iron-sequestering protein complex ferritin and abnormalities of the $B$ vitamins folic acid and vitamin $B_{12}$ [25]. The lack of association of these factors with CKD stage in the present results suggests that deficiencies of these factors do not cause renal anemia in CKD.

\section{Conclusion}

In conclusion, the current study showed that RBC lifespan tended to deteriorate with the progression of CKD, with significant deterioration already apparent in even the early stages of CKD. Meanwhile, although there may be a trend towards a temporary compensatory increase in EPO, we did not find evidence linking EPO deficiency to CKD progression. The present findings suggest that increased RBC destruction may be more important than other indicators in the etiology of renal anemia. 


\section{Acknowledgements}

We would like to thank Seekya Biotechnology Ltd., (Shenzhen, China) for lending us an ELS Tester CO breath test device and thank staff in the Department of Nephrology, Nanshan Hospital (Shenzhen, China) for assisting with alveolar air sample collection. We thank Ann Power Smith, PhD, of Write Science Right (www.writescienceright.com) for providing professional scientific language editing of the final manuscript.

\section{Statement of Ethics}

The protocol for the research project was approved by the Nanshan Hospital Ethics Committee and confirmed to conform with provisions of the Declaration of Helsinki (as revised in Tokyo 2004).

\section{Disclosure Statement}

The authors declare no conflicts of interest.

\section{Author Contribution}

All authors contributed significantly to this work and are in agreement with the content of the manuscript.

\section{References}

1 KDOQI; National Kidney Foundation. KDOQI Clinical Practice Guidelines and Clinical Practice Recommendations for Anemia in Chronic Kidney Disease. Am J Kidney Dis. 2006 May;47(5 Suppl 3):S11-145.

2 Sato Y, Yanagita M. Renal anemia: from incurable to curable. Am J Physiol Renal Physiol. 2013 Nov; 305(9):F1239-48.

3 Tsagalis G. Renal anemia: a nephrologist's view. Hippokratia. 2011 Jan;15 Suppl 1:39-43.

4 Besarab A, Bolton WK, Browne JK, Egrie JC, Nissenson AR, Okamoto DM, et al. The effects of normal as compared with low hematocrit values in patients with cardiac disease who are receiving hemodialysis and epoetin. $\mathrm{N}$ Engl J Med. 1998 Aug;339(9):584-90.

5 Singh AK, Szczech L, Tang KL, Barnhart H, Sapp S, Wolfson M, et al.; CHOIR Investigators. Correction of anemia with epoetin alfa in chronic kidney disease. N Engl J Med. 2006 Nov;355(20):2085-98.

6 Pfeffer MA, Burdmann EA, Chen CY, Cooper ME, de Zeeuw D, Eckardt KU, et al.; TREAT Investigators. A trial of darbepoetin alfa in type 2 diabetes and chronic kidney disease. N Engl J Med. 2009 Nov;361(21):2019-32.

7 Aapro M, Jelkmann W, Constantinescu SN, Leyland-Jones B. Effects of erythropoietin receptors and erythropoiesis-stimulating agents on disease progression in cancer. Br J Cancer. 2012 Mar;106(7):1249-58.

8 Joske RA, McAlister JM, Prankerd TA. Isotope investigations of red cell production and destruction in chronic renal disease. Clin Sci. 1956 Nov;15(4):511-22.

9 Loge JP, Lange RD, Moore CV. Characterization of the anemia associated with chronic renal insufficiency. Am J Med. 1958 Jan;24(1):4-18.

10 Strocchi A, Schwartz S, Ellefson M, Engel RR, Medina A, Levitt MD. A simple carbon monoxide breath test to estimate erythrocyte turnover. J Lab Clin Med. 1992 Sep;120(3):392-9.

11 Furne JK, Springfield JR, Ho SB, Levitt MD. Simplification of the end-alveolar carbon monoxide technique to assess erythrocyte survival. J Lab Clin Med. 2003 Jul;142(1):52-7.

12 Zhang HD, Ma YJ, Liu QF, Ye TZ, Meng FY, Zhou YW, et al. Human erythrocyte lifespan measured by Levitt's CO breath test with newly developed automatic instrument. J Breath Res. 2018 Mar;12(3):036003.

13 Loge JP, Lange RD, Moore CV. Characterization of the anemia associated with chronic renal insufficiency. Am J Med. 1958 Jan;24(1):4-18.

14 Desforges JF, Dawson JP. The anemia of renal failure. AMA Arch Intern Med. 1958 Feb;101(2):326-32. 
15 Ly J, Marticorena R, Donnelly S. Red blood cell survival in chronic renal failure. Am J Kidney Dis. 2004 Oct; 44(4):715-9.

16 Marcelli D, Bayh I, Merello JI, Ponce P, Heaton A, Kircelli F, et al. Dynamics of the erythropoiesis stimulating agent resistance index in incident hemodiafiltration and high-flux hemodialysis patients. Kidney Int. 2016 Jul; 90(1):192-202.

17 Panichi V, Scatena A, Rosati A, Giusti R, Ferro G, Malagnino E, et al. High-volume online haemodiafiltration improves erythropoiesis-stimulating agent (ESA) resistance in comparison with low-flux bicarbonate dialysis: results of the REDERT study. Nephrol Dial Transplant. 2015 Apr;30(4):682-9.

18 Ahmed MS, Langer H, Abed M, Voelkl J, Lang F. The uremic toxin acrolein promotes suicidal erythrocyte death. Kidney Blood Press Res. 2013;37(2-3):158-67.

19 Ahmed MS, Abed M, Voelkl J, Lang F. Triggering of suicidal erythrocyte death by uremic toxin indoxyl sulfate. BMC Nephrol. 2013 Nov; 14(1):244.

20 Lang F, Bissinger R, Abed M, Artunc F. Eryptosis - the neglected cause of anemia in end stage renal disease. Kidney Blood Press Res. 2017;42(4):749-60.

21 Macdougall IC. Role of uremic toxins in exacerbating anemia in renal failure. Kidney Int Suppl. 2001 Feb; 78:S67-72.

22 Shima H, Miya K, Okada K, Minakuchi J, Kawashima S. Sucroferric oxyhydroxide decreases serum phosphorus level and fibroblast growth factor 23 and improves renal anemia in hemodialysis patients. BMC Res Notes. 2018 Jun;11(1):363.

23 Eschbach JW. The anemia of chronic renal failure: pathophysiology and the effects of recombinant erythropoietin. Kidney Int. 1989 Jan;35(1):134-48.

24 Artunc F, Risler T. Serum erythropoietin concentrations and responses to anaemia in patients with or without chronic kidney disease. Nephrol Dial Transplant. 2007 Oct;22(10):2900-8.

25 Hutchinson FN, Jones WJ. A cost-effectiveness analysis of anemia screening before erythropoietin in patients with end-stage renal disease. Am J Kidney Dis. 1997 May;29(5):651-7. 